\title{
An Efficient Synthesis of 4'-Vinylated Carbocyclic Nucleoside Analogues via Two Directional Ring-closing Metathesis
}

\author{
Hua Li and Joon Hee Hong \\ BK21-Project Team, College of Phamacy, Chosun Lnwersitw, Gwangin 501-759, Korea. E-mail honghachosunac.kr \\ Received January 30,2008
}

Two directional ring-closing metathesis (RCM) was applied successfully to the sy'nthesis of 4 -vinylated carbocyclic micleoside analogues from the trivinyl intermediate 12. which was readily made using a sequential Claisen rearrangement and ring-closing metathesis (RCM) starting from Weinreb amide 5. An antiviral evaluation of the syinthesized compounds against various vinuses such as HIV. HSV-I. HSV-2 and HCMV revealed that the gianine analogue 20 have moderate anti-HIV activity in the MT-4 cell line ( $E C_{510}=10.2 \mu \mathrm{M}$ ).

Key Words : Vinylated carbocyclic nucleoside. Antiviral agents. Weinreb amide

\section{Introduction}

Recently. several branched nucleosides ${ }^{l}$ have been synthesized and evaluated as potent antitumor or antiviral agents. Among them. $4^{\prime} \alpha$-ethenyl and $4^{\prime} \alpha$-ethyy dine analogues $1^{2}$ and $2^{3}$ which have an additional double or triple bond at the 4'-position. were reported to have potent antiviral and antitumor activities (Figure 1). Carbocyclic nucleosides ${ }^{4}$ are a group of compounds that are structurally' similar to natural nucleosides. in which the furanose oxygen is replaced by a methylene group. Replacement of the furanose ring oxy'gen by carbon is of particular interest because the resulting carbocyclic nucleosides show greater metabolic stability to phosphorylase. which cleaves the glycosidic bond of nucleosides. The recent discovery of carbovir $3^{6}$ and abacavir $4^{7}$ as anti-HIV agents has increased interest in the syinthesis of novel nucleosides in this class of compounds.

Stimulated by these interesting molecular structures and their antiviral activity relationship. in this study a novel class of carbocyclic nucleosides containing 4'-electron-rich branch such as vinyl group was synthesized.

Weinreb amide 5. which was the starting material. was readily synthesized from the commercially available ethyl

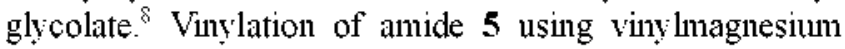
bromide gave the alkyl vinyl ketone derivative 6 . Compound 6 was subjected to Homer-Wadsworth-Emmons (HWE) reaction conditions ${ }^{y}$ to provide $\alpha . \beta$-unsaturated ethyl ester 7 as an $E / Z$ isomeric mixture. It was unnecessary to separate

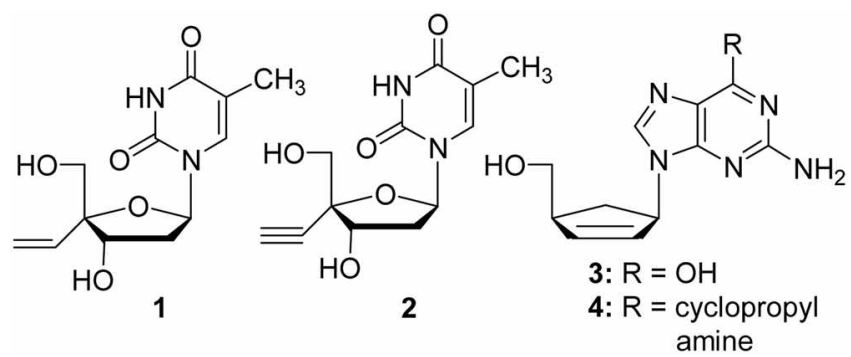

Figure 1. Structures of potent nucleosides as antiviral agents. the isomers because they were merged into a single isomer in the subsequent reaction. Ester 7 was reduced to allylic alcohol 8 using diisobutylaluminum hydride. which underwent a [3.3]-sigmatropic rearrangement ${ }^{10}$ using triethyl orthoacetate to give a $\gamma . \delta$-unsaturated ester 9 . The direct conversion of ester 9 to aldehyde 10 was made possible by the slow addition of DIBALH to the reaction mixture in toluene solvent system at $-78^{\circ} \mathrm{C}$. Aldehyde 10 was subjected to carbonyl addition by $\mathrm{CH}_{2}=\mathrm{CHMgBr}$ to yield trivinyl derivative 11 (Scheme 1).

Two directional cyclization of trivinyl 11 was perfomed under standard ring-closing metathesis conditions ${ }^{11}$ using a $2^{\text {nd }}$ generation Grubbs catalyst ${ }^{9}$ to provide cyclopentenols $12 \alpha$ and $12 \beta$ respectively. The relative stereochemical assignments were determined by proton NOE experiments. Upon irradiation of $\mathrm{C}_{1}-\mathrm{H}$, relatively strong NOE was observed at the methylene protons of the hydroxymethyl group $12 \alpha$ but not at the methylene protons of $12 \dot{\beta}$ (Figure

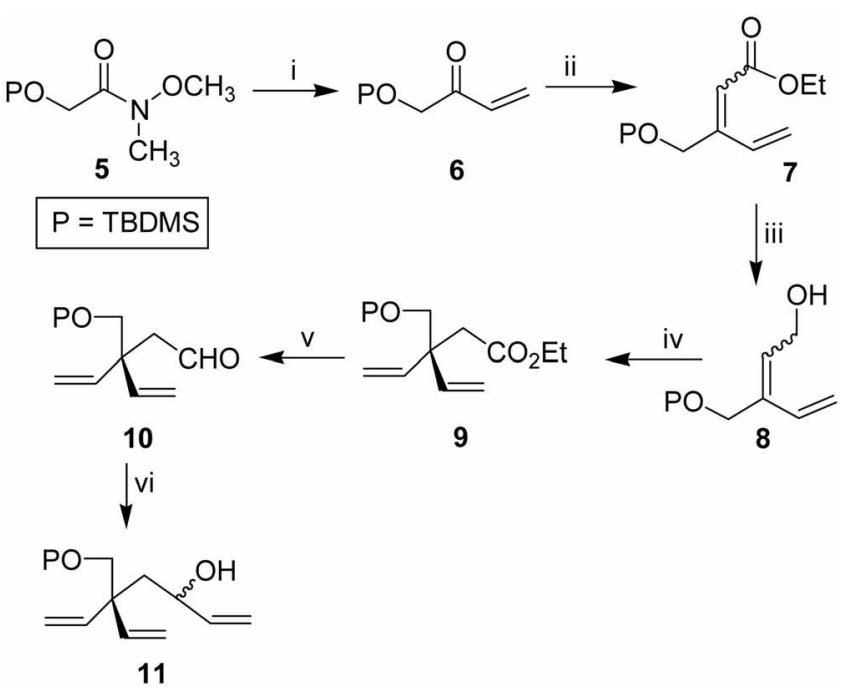

Scheme 1. Synthesis route of trivinyl intemediate 11. Reagents: ij vinylmagnesium bromide, THF, 0 " $\mathrm{C}$; ii) triethylphosphonoacetale. $\mathrm{NaH}$, THF: iii) DIBALH, $\mathrm{CH}_{2} \mathrm{Cl}_{2}$; iv) triethylorthoacetate, propionic acid o vemight, $135-140^{\circ} \mathrm{C}$; v) DIBALH, toluene, $-78^{\circ} \mathrm{C}$; vi) viny lnagnesium bromide, $\mathrm{THF}_{\mathrm{z}}-78^{\circ} \mathrm{C}$. 


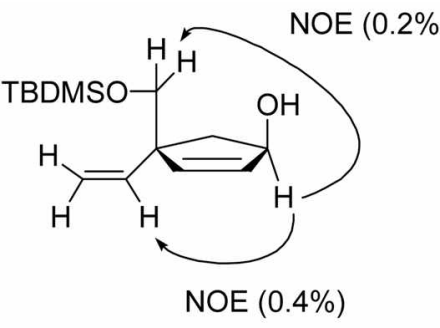

$12 \beta$

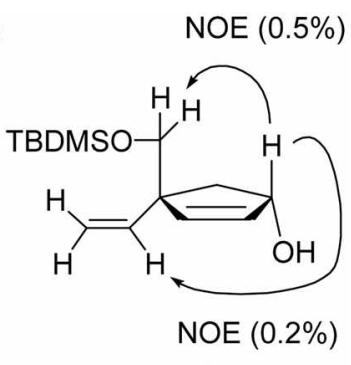

$12 \alpha$
Figure 2. NOE comparisons of compounds $12 \alpha$ and $12 \beta$.

2).

Initially. an attempt was made to synthesize the target compounds via mesylation and nucleophilic substitution from $12 \alpha$. However. the reaction produced a very low yield and was not reproducible. In order to couple the nucleosidic bases (adenine. cytosine, 2-amino-6-chloropurine) to ally lic derivative $12 \beta$ using well known palladium( $(0)$-catalysis, ${ }^{13}$ cyclopentenol $12 \beta$ was transformed to the allylic formate analogue 13 using ethyl chlorofornate. Compound 13 was coupled with the nucleosidic base anions generated by $\mathrm{NaH} /$ DMSO using a catalyst [tris(dibenzylidene-acetone)-dipalladium(0)-chloroform] adduct to provide the carbocyclic nucleoside analogues 1+-16. Removal of the silyl protecting group from them was preformed by treating the compounds with tetrabutylammonium fluoride (TBAF) to give nucleosides 17-19. Treatment of compound 19 with 2-mercaptoethanol and sodium methoxide in methanol. followed by neutralization with acetic acid gave the desired guanine carbocyclic nucleoside analogie 20 (Scheme 2).

All the synthesized compounds $17 \sim 20$ were tested against several viruses such as HIV-1 (MT- 4 cells). HSV-1 and HSV-2 (CCL 18 cells), and HCMV (AD-169). ${ }^{13}$ As shown in Table I. some of the compounds showed antiviral activity. In particular. the guanine nucleoside analogue 20. exhibited moderate anti-HIV activity in MT-4 cells $\left(\mathrm{EC}_{\text {Si }}=10.2 \mu \mathrm{M}\right)$. It is believed that the arrangement in the carbocyclic guanine

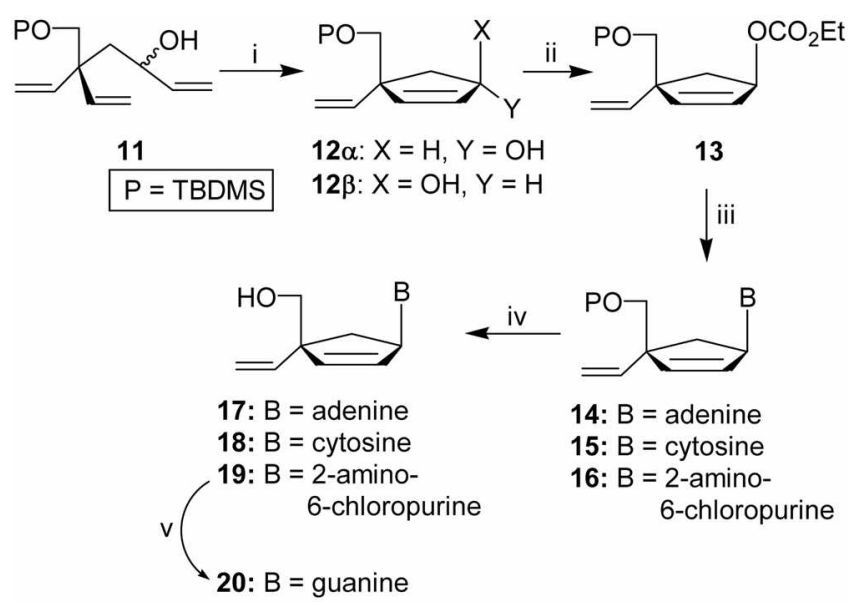

Scheme 2. Synthesis route of taregt nucleosides. Reagents: i) Grubbs catalyst (II), $\mathrm{CH}_{2} \mathrm{Cl}_{2} ;$ ii) $\mathrm{ClCO}_{2} \mathrm{Et}$, prridine, DMAP; iii) nucleosidic bases, $\mathrm{Pd}_{2}(\mathrm{dba})_{2} \mathrm{CHCl}_{3}, \mathrm{P}(\mathrm{O}-i-\mathrm{Pr})_{2}, \mathrm{NaH}, \mathrm{THF} / \mathrm{DMSO}$; iv) TBAF, THF; v) (a) 2-mercaptoethanol, $\mathrm{NaOMe}, \mathrm{MeOH}$, (b) $\mathrm{CH}_{3} \mathrm{COOH}$.
Table 1. The antiviral activity of the synthesized compounds

\begin{tabular}{|c|c|c|c|c|c|}
\hline & $\begin{array}{c}\text { HIV-1 } \\
\mathrm{EC}_{50}(\mu \mathrm{M})\end{array}$ & $\begin{array}{l}\text { HSV-I } \\
\text { EC }_{s o}(\mu M)\end{array}$ & $\begin{array}{c}\text { HSV-2 } \\
\mathrm{EC}_{\mathrm{s}_{0}}(\mu \mathrm{M})\end{array}$ & $\begin{array}{c}\text { HCMV } \\
\mathrm{EC}_{5 \sigma}(\mu \mathrm{M})\end{array}$ & $\begin{array}{l}\text { cytotoxicity } \\
\mathrm{C}_{5 \sigma}(\mu \mathrm{M})\end{array}$ \\
\hline 17 & 95 & $>100$ & $>100$ & $>100$ & 95 \\
\hline 18 & 77.9 & $>100$ & 90.5 & 23.7 & $>100$ \\
\hline 19 & $>100$ & $>100$ & $>100$ & 61.2 & $>100$ \\
\hline 20 & 10.2 & 65.8 & $>100$ & 41.5 & 99 \\
\hline AZT & 0.01 & $N D$ & $N D$ & ND & 1.15 \\
\hline $\mathrm{GCV}$ & $\mathrm{ND}$ & ND & ND & 0.8 & $>10$ \\
\hline $\mathrm{ACV}$ & $N D$ & 0.2 & ND & $N D$ & $>100$ \\
\hline
\end{tabular}

AZT: Azidothymidine: GCV: Ganciclovir: ACV: Acyclotir: ND: Not Determined. $\mathrm{EC}_{S n}(\mu \mathrm{M})$ : Concentration required to inhibit $50^{\circ}, 0$ of the virus induced crtopathicity: $\mathrm{CC}_{5 \cup}(\mu \mathrm{M})$ : Concentration required to reduce the cell viability by $50^{\circ}$.

nucleoside analogue 20 may be conformationally similar to that in natural nucleosides containing ribose. Hence, this arrangement will enlance the level of phosphorylation by kinase to produce the active monophosphate form. This suggests that this class of 4 '-vinylated carbocyclic $N$-nucleoside. which has no hydrosy group in the 3 '-position, can be a novel structural template for the development of new antiviral agents.

In summary. a convenient method for synthesizing 4'vinylated carbocyclic nucleoside analogues via two directional RCM from the Weinreb amide was developed. Based on this strategy. the syntheses of other nucleosides such as acetylated carbocyclic nucleosides with different nucleobases are currently underway.

\section{Experimental Section}

The melting points were determined on a Mel-temp II laboratory device and are uncorrected. The NMR spectra were recorded on a JEOL 300 Fourier transform spectrometer. The chemical shifts are reported in parts per million $(\delta)$ and the signals are quoted as s (singlet), d (doublet). $\mathrm{t}$ (triplet). q (quartet). $\mathrm{m}$ (multiplet) and dd (doublet of doublets). The UV spectra were obtained on a Beckman DU-7 spectrophotometer. The elemental analyses were performed using a Perkin-Elmer 2400 analyzer (Perkin-Elmer. Norwalk. CT, USA). TLC was performed on Uniplates (silica gel) purchased from Analtech Co. Unless specified otherwise, all reactions were carried out in a $\mathrm{N}_{2}$ atmosphere. Dry dichloromethane. benzene and pyridine were obtained by distillation from $\mathrm{CaH}_{\beth}$. Dry THF was obtained by distillation from $\mathrm{Na}$ and benzophenone inmediately before use.

2-(tert-Butyldimethylsilyloxy)-1-vinyl-ethanone (6). Vinylmagnesium bromide (18.0 mL. $1.0 \mathrm{M}$ solution in THF) was added slowly to a solution of Weinreb amide $5(3.5 \mathrm{~g}$. $14.99 \mathrm{mmol})$ in dry THF $(70 \mathrm{~mL})$ at $0{ }^{\circ} \mathrm{C}$. After $5 \mathrm{~h}$, a saturated $\mathrm{NH}_{4} \mathrm{Cl}$ solution ( $18 \mathrm{~mL}$ ) was added, and the reaction mixture was warmed slowly to rt. The mixture was extracted with EtOAc $(2 \times 100 \mathrm{~mL})$. The combined organic layer was dried over $\mathrm{MgSO}_{4}$. filtered. and evaporated. The residue was purified by silica gel column chromatography (EtOAc/hexane. 1:10) to give compound 6(2.07 g. 69\%) as 
a colorless oil: ${ }^{1} \mathrm{H}$ NMR $\left(\mathrm{CDCl}_{3} .300 \mathrm{MHz}\right) \delta 6.53$ (dd. $J=$ $17.7,10.8 \mathrm{~Hz} . \mathrm{lH}) .6 .27(\mathrm{~d}, J=17.4 \mathrm{~Hz} .1 \mathrm{H}), 5.67(\mathrm{~d}, J=$ $10.5 \mathrm{~Hz}, 1 \mathrm{H}$ ) 4.27 (s. $2 \mathrm{H}$ ). 0.83 (s. $9 \mathrm{H}) .0 .01$ (s. $6 \mathrm{H}$ ) ${ }^{13} \mathrm{C}$ NMR $\left(\mathrm{CDCl}_{3}\right) \delta 198.78,131.67 .128 .87,68.56 .25 .76$. 18.34. -5.47

(E) and (Z)-4-(tert-Butyldimethylsilyloxy)-3-vinyl-but2-enoic acid ethyl ester (7). Triethyl phosphonoacetate ( $3.73 \mathrm{~g}, 16.64 \mathrm{~mm}$ ol) was added dropwise to a suspension of sodium hydride $(0.4 \mathrm{~g} .16 .64 \mathrm{mmnol})$ in distilled THF (80 $\mathrm{mL}$ ) at $0^{\circ} \mathrm{C}$. The nixture was stirred at room temperature for $1 \mathrm{~h}$. The ketone $6(3.33 \mathrm{~g}, 16.64 \mathrm{mmol})$ was then added to this mixture and the mixture was stirred for $2 \mathrm{~h}$. The solution was neutralized with $\mathrm{AcOH}(3.5 \mathrm{~mL})$. poured into $\mathrm{H}_{2} \mathrm{O}(\mathrm{I} 20$ $\mathrm{mL}$ ) and extracted with EtOAc $(120 \mathrm{~mL} \times 2)$. The combined organic layer was washed with brine. dried over anhydrous $\mathrm{MgSO}_{4}$, filtered and evaporated. The residue was purified by silica gel column chromatography (EtOAc/hexane. 1:12) to give compound $7(3.28 \mathrm{~g} .73 \%)$ as a colorless oil: ${ }^{1} \mathrm{H}$ NMR $(\mathrm{CDCl}, 300 \mathrm{MHz}) \delta 5.78$ (dd. $J=17.1,10.5 \mathrm{~Hz}, \mathrm{lH}), 5.30$ (dd. $J=16.2 .0 .9 \mathrm{~Hz}, 1 \mathrm{H}) .5 .26(\mathrm{~d}, J=16.0 \mathrm{~Hz}, 1 \mathrm{H}), 5.20(\mathrm{~s}$. lH), $4.22(\mathrm{t}, J=7.0 \mathrm{~Hz} .2 \mathrm{H}$ ). 4.12 (s. $2 \mathrm{H}) .4 .05$ (q. $J=6.9$ Hz, 2H). 1.19 (t. $J=6.8 \mathrm{~Hz} .3 \mathrm{H}), 0.84$ (s. 9H), $0.02(\mathrm{~m}, 6 \mathrm{H})$.

$(E)$ and $(Z)$-4-(tert-Butyldimethylsilyloxy)-3-vinyl-but2-en-1-ol (8). DIBALH (31.08 mL, 1.0 M solution in hexane) was added slowly to a solution of compound 7 (4.0 g. $14.8 \mathrm{mmol})$ in $\mathrm{CH}_{2} \mathrm{Cl}_{2}(120 \mathrm{~mL})$ at $-20^{\circ} \mathrm{C}$, and stirred for $2 \mathrm{~h}$ at the same temperature. Methanol $(30 \mathrm{~mL})$ was then added to this mixture. The mixture was stirred at room temperature for $2 \mathrm{~h}$, and the resulting solid was filtered through a Celite pad. The filtrate was concentrated under vacuum and the residue was purified by silica gel columm chromatography (EtOAc/hexane. 1:5) to give alcohol 8 $(2.97 \mathrm{~g} .88 \%)$ as a colorless oil: ${ }^{1} \mathrm{H}$ NMR $\left(\mathrm{CDCl}_{3}, 300 \mathrm{MHz}\right)$ $\delta 5.78(\mathrm{~s}, \mathrm{lH}) .5 .59$ (dd. $J=16.2 .10 .2 \mathrm{~Hz} . \mathrm{lH}) .5 .4 \mathrm{l}(\mathrm{d} . J=$ $16.2 \mathrm{~Hz}, \mathrm{IH}), 5.19$ (d. $J=10.2 \mathrm{~Hz}, 1 \mathrm{H}), 4.22(\mathrm{t} . J=6.8 \mathrm{~Hz}$. $2 \mathrm{H}), 4.18$ (s. $2 \mathrm{H}), 0.83(\mathrm{~m}, 9 \mathrm{H}) .0 .02(\mathrm{~m} .6 \mathrm{H})$.

( \pm -3-( $t$-Butyldimethylsilyloxymethyl)-3-vinyl-pent-tenoic acid ethyl ester (9). A solution of allylic alcohol 8 $(5.2 \mathrm{~g} .22 .76 \mathrm{mmol})$ in triethyl orthoacetate $(90 \mathrm{~mL})$ and 0.2 $\mathrm{mL}$ of propionic acid was heated overnight at $135-140^{\circ} \mathrm{C}$ with constant stirring under the conditions for the distillative removal of ethanol. The excess triethyl orthoacetate was distilled off and the residue was purified by silica gel column chromatography (EtOAc/hexane. 1:20) to give compound 9 $\left(5.36\right.$ g. $79 \%$ ) as a colorless oil: ${ }^{1} \mathrm{H} \mathrm{NMR}\left(\mathrm{CDCl}_{3}, 300 \mathrm{MHz}\right)$ $\delta 5.92-5.87$ (m. $2 \mathrm{H}$ ), 5.79 (m. $2 \mathrm{H}$ ). $5.10-5.04$ (n. $2 \mathrm{H}$ ). $4.05-$ $3.95(\mathrm{~m} .4 \mathrm{H}) .2 .83(\mathrm{~d} . J=4.2 \mathrm{~Hz} .2 \mathrm{H}) .1 .20(\mathrm{t} . J=7.2 \mathrm{~Hz}$. $3 \mathrm{H}), 0.82$ (s. $9 \mathrm{H}), 0.01$ (s. $6 \mathrm{H}):{ }^{13} \mathrm{C}$ NMR $\left(\mathrm{CDCl}_{3}\right) \delta 171.71$. $1+7.61,1+2.98,116.54 .111 .49 .68 .98 .60 .38,48.21,39.56$. $25.77,18.49,14.21,-5.56$.

( \pm )-3-( $t$-Butyldimethylsilyloxymethyl)-3-vinyl-pent-4enal (10). DIBALH (6.1 mL, $1.5 \mathrm{M}$ solution in toluene) was added slowly to a solution of compound 9 ( 2.5 g. 8.37 mmol) in toluene $(40 \mathrm{~mL})$ at $-78{ }^{\circ} \mathrm{C}$. and stirred for 10 minutes at the same temperature. Methanol $(7 \mathrm{~mL})$ was then added to this mixture. The resulting mixture was stirred at room temperature for $1 \mathrm{~h}$. and the resulting solid was filtered through a Celite pad. The filtrate was concentrated under vacuum and the residue was purified by silica gel column chromatograply (EtOAc/hexane, 1:15) to give compound $10(1.45 \mathrm{~g} .68 \%)$ as a colorless oil: ${ }^{1} \mathrm{H}$ NMR $\left(\mathrm{CDCl}_{3} .300\right.$ MHz) $\delta 9.69(\mathrm{~s} .1 \mathrm{H}), 5.90-5.82(\mathrm{~m}, 2 \mathrm{H}) .5 .75-5.67(\mathrm{~m} .2 \mathrm{H})$, $5.11 .5 .04(\mathrm{~m}, 2 \mathrm{H}), 3.99$ (dd. $J=12.8,8.8 \mathrm{~Hz} .2 \mathrm{H}), 2.90(\mathrm{dd}$, $J=10.8,4.2 \mathrm{~Hz}, 2 \mathrm{H}$ ), 0.84 (s. $9 \mathrm{H}), 0.02$ (s. $6 \mathrm{H}) ;{ }^{13} \mathrm{C}$ NMR $\left(\mathrm{CDCl}_{3}\right) \delta 202.87,147.54,143.04,117.19,112.65 .69 .25$, 48.87. 39.56.25.59. 18.74. -5.61 .

$( \pm)$-5-( $(t$-Butyldimethylsilyloxymethyl)-5-vinyl-hepta1,6-dien-3-ol (11): Vinylmagnesium bromide (18.86 mL, $1.0 \mathrm{M}$ solution in THF) was added slowly to a solution of compound $10(4.0 \mathrm{~g} .15 .72 \mathrm{mmol})$ in dry THF $(60 \mathrm{~mL})$ at $-78^{\circ} \mathrm{C}$. After $5 \mathrm{~h}$. a saturated $\mathrm{NH}_{4} \mathrm{Cl}$ solution $(20 \mathrm{~mL})$ and water $(100 \mathrm{~mL})$ was then added, and the reaction mixture was slowly warmed to $\mathrm{rt}$. The mixture was extracted with EtOAc $(2 \times 120 \mathrm{~mL})$. The combined organic layer was dried over $\mathrm{MgSO}_{4}$. filtered. and evaporated. The residue was purified by silica gel colunn cluromatography (EtOAc/hexane, 1:15) to give compound $11(3.37 \mathrm{~g} .76 \%)$ as a colorless oil: ${ }^{1} \mathrm{H}$ NMR $\left(\mathrm{CDCl}_{3} .300 \mathrm{MHz}\right) \delta 6.04-5.72(\mathrm{~m}, 3 \mathrm{H}), 5.26-4.95$ $(\mathrm{m}, 3 \mathrm{H}) .5 .12-5.05(\mathrm{~m} .3 \mathrm{H}), 3.65(\mathrm{~m} .3 \mathrm{H}), 1.67 \cdot 1.54(\mathrm{~m}$, $2 \mathrm{H}) .0 .83(\mathrm{~m}, 9 \mathrm{H}), 0.02(\mathrm{~m} .6 \mathrm{H})$.

$(r e l)-(1 R, 4 S)-4-(t$-Butyldimethylsilyloxymethyl)-t-vinyl-cyclopent-2-enol (12 $\beta)$; and $(r e t)-(1 S, 4 S)-t-(t-B u t y l-$ dimethylsilyloxymethyl)-t-vinyl-cyclopent-2-enol $(12 c)$. $2^{\mathrm{nt} l}$ generation Grubbs catalyst $(152 \mathrm{mg} .0 .18 \mathrm{mmol})$ was added to a solution of compound $11(1.56 \mathrm{~g} .5 .54 \mathrm{mmol})$ in dry $\mathrm{CH}_{2} \mathrm{Cl}_{2}(15 \mathrm{~mL})$. The reaction mixture was refluxed overnight. and cooled to room temperature. The mixture was concentrated under vacuum, and the residue was purified by silica gel colunn chromatography (EtOAc/hexane, 1:12) to give cyclopentenol $12 \beta(549 \mathrm{mg} .39 \%$ ) and $12 \alpha(535 \mathrm{mg}$. $38 \%$ ) as colorless oils, respectively. Cyclopentenol $12 \beta .{ }^{\circ} \mathrm{H}$ NMR $\left(\mathrm{CDCl}_{3}, 300 \mathrm{MHz}\right) \delta 6.11-6.03(\mathrm{~m} .3 \mathrm{H}) .5 .90-5.8 \mathrm{l}(\mathrm{m}$, $3 \mathrm{H}) .4 .87$ (dd. $J=7.4 .1 .2 \mathrm{~Hz}, \mathrm{lH}) .3 .54$ (s. $2 \mathrm{H}) .2 .65$ (dd, $J$ $=13.4 .7 .0 \mathrm{~Hz} . \mathrm{lH}) .1 .85(\mathrm{dd} . J=13.4,2.8 \mathrm{~Hz}, 1 \mathrm{H}) .0 .8 \mathrm{l}(\mathrm{s}$, 9H). 0.01 (s. 6H): ${ }^{13} \mathrm{C}$ NMR $\left(\mathrm{CDCl}_{3}\right) \delta 145.29 .143 .59$. $140.49,133.98,75.34,69.78,57.82,46.78,25.45,18.67$. -5.50 .

Cyclopentenol $12 \alpha .{ }^{1} \mathrm{H}$ NMR $\left(\mathrm{CDCl}_{3}, 300 \mathrm{MHz}\right) \delta 6.13-$ $6.06(\mathrm{~m}, 3 \mathrm{H}), 5.92-5.84(\mathrm{~m}, 3 \mathrm{H}), 4.85(\mathrm{dd}, J=5.6 .1 .4 \mathrm{~Hz}$. $1 \mathrm{H}) .3 .56$ (s. $2 \mathrm{H}$ ). $2.6 \mathrm{l}$ (dd. $J=13.2 .7 .2 \mathrm{~Hz}, 1 \mathrm{H}$ ). 1.86 (dd. $J$ $=13.2 .3 .3 \mathrm{~Hz}, \mathrm{lH}$ ). 0.83 (s. 9H). $0.02(\mathrm{~s} .6 \mathrm{H}):{ }^{13} \mathrm{C}$ NMR $\left(\mathrm{CDCl}_{3}\right) \delta 145.72 .142 .51 .141 .79,132.29,74.29,68.49$. 58.39. 44.28.25.71. 18.59. -5.57 .

(rel)-(1R,4S)-1-Ethoxy carbonyloxy-4-( $t$-butyldimethylsilyloxymethyl)-4-vinyl-cyclopent-2-ene (13): Ethyl chloroformate (1.87 mL, $17.3 \mathrm{mmol}$ ) and DMAP ( $85 \mathrm{mg}$. $0.7 \mathrm{mmol})$ were added to a solution of $12 \beta(2.2 \mathrm{~g} .8 .65$ munol) in andydrous pyridine $(15 \mathrm{~mL})$. The reaction mixture was stirred overnight at room temperature. The reaction mixture was quenched with a saturated $\mathrm{NaHCO}_{3}$ solution $(2.0 \mathrm{~mL})$ and concentrated under vacuum. The residue was extracted with $\mathrm{EtOAc} / \mathrm{H}_{2} \mathrm{O}$, and the organic layer was dried over $\mathrm{MgSO}_{4}$, filtered, and concentrated. The residue was purified by silica gel column cluromatography (EtOAc/ 
hexane. 1:10) to give compound $13(1.97 \mathrm{~g}, 70 \%)$ as a colorless syrup: ${ }^{1} \mathrm{H}$ NMR $\left(\mathrm{CDCl}_{3}, 300 \mathrm{MHz}\right) \delta 6.46(\mathrm{~d}, J=$ $5.6 \mathrm{~Hz} . \mathrm{lH}$ ). $6.37-6.29$ (m. $2 \mathrm{H}), 6.09-5.94$ (m. $2 \mathrm{H}$ ), 5.75 (d. $J$ $=4.6 \mathrm{~Hz}, 1 \mathrm{H}), 4.38(\mathrm{q}, J=7.4 \mathrm{~Hz} .2 \mathrm{H}), 3.89(\mathrm{~d}, J=9.2 \mathrm{~Hz}$. $1 \mathrm{H}), 3.81$ (d. $J=9.0 \mathrm{~Hz}, 1 \mathrm{H}), 2.61$ (dd, $J=13.6,6.8 \mathrm{~Hz}$. lH), $2.33(\mathrm{dd}, J=13.6 .3 .6 \mathrm{~Hz}, \mathrm{IH}), 1.42(\mathrm{t} . J=7.4 \mathrm{~Hz} .3 \mathrm{H})$. 0.83 (s. 9H). 0.01 (s. $6 \mathrm{H}$ ): ${ }^{13} \mathrm{C}$ NMR $\left(\mathrm{CDCl}_{3}\right) \delta 155.21$. 145.65. 142.42. 140.54, 124.67, 82.54. 71.49. 63.88, 57.32. $40.96 .25 .61,18.70,14.51,-5.60$.

(rel)-(1'R, ' $S$ )-9-[4-(t-Butyldimethylsilyloxymethyl)-tvinyl-cyclopent-2-en-1-yl] adenine (14). Adenine (79.45 mg. $0.588 \mathrm{mmol}$ ) was added to a pure $\mathrm{NaH}(14.4 \mathrm{mg} .0 .588$ mnol) in anlydrous DMSO $(3.6 \mathrm{~mL})$. The reaction mixture was stirred for $30 \mathrm{~min}$ at $50-55^{\circ} \mathrm{C}$ and cooled to room temperature. At the same time, $\mathrm{P}(\mathrm{O}-\mathrm{i}-\mathrm{Pr})_{3}(0.0576 \mathrm{~mL} .0 .132$ mmol) was added to a solution of $\mathrm{Pd}_{2}(\mathrm{dba})_{3} \cdot \mathrm{CHCl}_{3}(2.76$ mg. $1.5 \mathrm{mmol})$ in anhydrous THF $(3.0 \mathrm{~mL})$, and stirred for 30 min. The catalyst solution in THF and compound $\mathbf{1 3}$ (172.4 mig. $0.528 \mathrm{mmol}$ ) dissolved in anlydrous THF $(3.0$ $\mathrm{mL}$ ) were added sequentially to the nucleosidic base solution of DMSO. The reaction mixture was heated and stirred ovemight under reflux. and quenched with water (1.2 $\mathrm{mL}$ ). The reaction solvent was removed under vacuunl. The residue was purified by silica gel column cluromatography (MeOH/EtOAc/Hexane. 0.1:1:2) to give compound 1+ (76.5 mg. $39 \%$ ) as a white solid: ${ }^{1} \mathrm{H}$ NMR $\left(\mathrm{CDCl}_{3}, 300 \mathrm{MHz}\right) \delta$ 8.33 (s. $1 \mathrm{H}), 8.01$ (s. $1 \mathrm{H}), 6.42$ (d. $J=5.4 \mathrm{~Hz}, \mathrm{IH}), 6.30-6.22$ (m, 2H). 6.07.5.95 (m. 2H), 5.77 (dd, $J=6.6 .1 .2 \mathrm{~Hz} . \mathrm{IH}$ ). 3.87 (d. $J=9.2 \mathrm{~Hz}, \mathrm{IH}) .3 .80(\mathrm{~d}, J=9.2 \mathrm{~Hz}, \mathrm{IH}) .2 .90$ (dd. $J$ $=13.4,8.4 \mathrm{~Hz}, 1 \mathrm{H}), 2.42(\mathrm{dd}, J=13.4 .6 .4 \mathrm{~Hz} .1 \mathrm{H}) .0 .82(\mathrm{~s}$. 9H), 0.01 (s. $6 \mathrm{H}):{ }^{13} \mathrm{C}$ NMR $\left(\mathrm{CDCl}_{3}\right) \delta 155.90 .152 .40$. $150.21,145.71 .142 .77,141.70 .140 .11 .125 .65 .118 .67$. 70.34. 60.01, 46.32. 42.61. 25.67, 18.71. -5.69: Anal. Calcd. for $\mathrm{C}_{1}, \mathrm{H}_{2}-\mathrm{N}_{5} \mathrm{OSi}: \mathrm{C}, 61.42 ; \mathrm{H}, 7.87: \mathrm{N}, 18.85$. Found: $\mathrm{C}$. $61.50 ; \mathrm{H}, 7.69 ;$ N. 18.91 .

(rel)-(1'R,t'S)-1-[4-(t-Butyldimethylsilyloxymethyl)-tvinyl-cyclopent-2-en-1-yl] cytosine (15). Compound 15 was synthesized from compound 13 using a similar procedure to that described for compound 14 y yield $44 \%:{ }^{1} \mathrm{H}$ NMR $\left(\mathrm{CDCl}_{3}, 300 \mathrm{MHz}\right) \delta 7.30(\mathrm{~d}, J=7.0 \mathrm{~Hz} . \mathrm{HH}), 6.44$ (dd. $J=5.4,1.2 \mathrm{~Hz}, 1 \mathrm{H}), 6.29-6.20(\mathrm{~m}, 2 \mathrm{H}) .6 .08-5.96(\mathrm{~m}$. $2 \mathrm{H}) .5 .76(\mathrm{dd} . J=6.6 .1 .4 \mathrm{~Hz}, 1 \mathrm{H}) .5 .54(\mathrm{~d} . J=7.0 \mathrm{~Hz}, 1 \mathrm{H})$. $3.85(\mathrm{~d} . J=9.4 \mathrm{~Hz}, 1 \mathrm{H}) .3 .79(\mathrm{~d} . J=9.2 \mathrm{~Hz}, 1 \mathrm{H}) .2 .91$ (dd. $J$ $=13.4 .8 .2 \mathrm{~Hz}, 1 \mathrm{H}), 2.44(\mathrm{dd} J=13.4 .6 .4 \mathrm{~Hz} .1 \mathrm{H}) .0 .81$ (s. 9H) 0.02 (s. $6 \mathrm{H}):{ }^{13} \mathrm{C}$ NMR $\left(\mathrm{CDCl}_{3}\right) \delta 165.41 .156 .78$. $145.90 .145 .31,142.20 .140 .72$. 123.61, 92.32, 69.68. 61.41. 47.78, 43.67, 25.40, 18.43, -5.48: Anal. Calcd. for $\mathrm{C}_{13} \mathrm{H}_{29} \mathrm{~N}_{3} \mathrm{O}_{2} \mathrm{Si}: \mathrm{C}, 62.21$ : H. 8.41; N, 12.09. Found: C. 62.31: $\mathrm{H}, 8.55 ; \mathrm{N}, 11.95$.

(rel)-(1'R,4'R)-9-[4-(t-Butyldimethylsilyloxymethyl)-4vinyl-cyclopent-2-en-1-yl]2-amino-6-chloropurine (16). Nucleoside analogue 16 was sytnthesized from compound 13 using a similar procedure to that described for compound 14 : yield $35 \%$ : ${ }^{1} \mathrm{H}$ NMR $\left(\mathrm{CDCl}_{3,300 \mathrm{MHz})} \delta 8.70(\mathrm{~s}, 1 \mathrm{H}), 6.38\right.$ (d, $J=5.4 \mathrm{~Hz} .1 \mathrm{H}), 6.25-6.18$ (m. $2 \mathrm{H}$ ). 6.02-5.95 (m, $2 \mathrm{H}$ ). $5.71(\mathrm{~d} . J=6.6 \mathrm{~Hz}, 1 \mathrm{H}) .3 .90(\mathrm{~d}, J=9.4 \mathrm{~Hz}, 1 \mathrm{H}) .3 .78$ (d. $J$ $=9.4 \mathrm{~Hz} .1 \mathrm{H}) .2 .90(\mathrm{dd} . J=13.6 .8 .4 \mathrm{~Hz} .1 \mathrm{H}) .2 .44(\mathrm{dd} . J=$
13.6. 6.6 Hz. 1H). $0.83(\mathrm{~s}, 9 \mathrm{H}), 0.01(\mathrm{~s}, 6 \mathrm{H}):{ }^{13} \mathrm{C}$ NMR $\left(\mathrm{CDCl}_{3}\right) \delta 159.56 .154 .88,152.51,145.32 .143 .71 .142 .90$, $141.43,132.77 .125 .43 .69 .99 .60 .31 .46 .54,42.60,25.67$, 18.79. -5.58; Anal. Calcd. for $\mathrm{C}_{19} \mathrm{H}_{28} \mathrm{ClN}_{5} \mathrm{OSi}: \mathrm{C} .56 .21: \mathrm{H}$, $6.95 ;$ N, 17.25 . Found: C. 56.11; H. 7.08: N, 17.12.

(rel)-(1'R,+'R)-9-[4-(Hydroxymethy])-t-viny]-cyclopent2-en-1-yl] adenine (17). TBAF $(0.54 \mathrm{~mL}$. $1.0 \mathrm{M}$ solution in THF) was added to a solution of compound 14 (126 mg. $0.362 \mathrm{mmol}) \mathrm{in}$ THF $(5 \mathrm{~mL})$ at $0^{\circ} \mathrm{C}$. The mixture was stirred overnight at room temperature. and concentrated. The residue was purified by silica gel column chromatography $\left(\mathrm{MeOH} / \mathrm{CH}_{2} \mathrm{Cl}_{2}, 1: 5\right)$ to give compound $17(61 \mathrm{mg} .73 \%$ ) as a white solid: mp $180-183^{\circ} \mathrm{C}$ : UV $\left(\mathrm{H}_{2} \mathrm{O}\right) \lambda_{\text {max }} 262.0 \mathrm{~nm}:{ }^{l} \mathrm{H}$ NMR (DMSO- $\left.c_{i}, 300 \mathrm{MHz}\right) \delta 8.37$ (s, 1H). 8.05 (s. 1H), 6.45 (dd. $J=5.4,1.2 \mathrm{~Hz} .1 \mathrm{H}$ ), 6.33-6.25 (m. 2H). 6.08-5.99 (m, $2 \mathrm{H}), 5.79$ (d. $J=6.8 \mathrm{~Hz} .1 \mathrm{H}) .4 .99(\mathrm{t} . J=5.4 \mathrm{~Hz}, \mathrm{lH}$ ), 3.83 (d. $J=9.2 \mathrm{~Hz} .1 \mathrm{H}), 3.72$ (d. $J=9.0 \mathrm{~Hz} .1 \mathrm{H}) .2 .92$ (dd. $J$ $=13.2,8.2 \mathrm{~Hz} .1 \mathrm{H}) .2 .48(\mathrm{dd} . J=13.2,6.2 \mathrm{~Hz} .1 \mathrm{H}){ }^{12} \mathrm{C}$ NMR (DMSO- $d_{6}$ ) $\delta 155.71 .152 .59 .151 .33 .145 .30 .144 .20$. $141.70,140.47,125.65 .119 .61 .69 .48,60.54 .46 .43,41.20$. Anal. Calcd. for $\mathrm{C}_{33} \mathrm{H}_{15} \mathrm{~N}_{5} \mathrm{O}: \mathrm{C}, 60.69: \mathrm{H}, 5.88 ;$ N. 27.22 Found: C, 60.80: H, 5.81; N, 27.32.

(rel)-(1'R,+'R)-1-[+-(Hydroxymethyl)-t-viny]-cyclopent2-en-1-yl] cytosine (18). Compound 18 was obtained from compound 15 using a similar procedure to that described for compound 17. yield $68 \%$; mp 160-163 $\mathrm{C}$. UV $\left(\mathrm{H}_{2} \mathrm{O}\right) \lambda_{\text {max }}$ $271.5 \mathrm{~mm} ;{ }^{1} \mathrm{H}$ NMR (DMSO- $\left.c_{6}, 300 \mathrm{MHz}\right) \delta 7.29$ (d. $J=6.9$ Hz. IH). 6.38 (d, $J=5.4 \mathrm{~Hz}, \mathrm{IH}$ ). 6.20-6.08 (m. $4 \mathrm{H}), 5.79$ (d. $J=6.6 \mathrm{~Hz} . \mathrm{lH}), 5.56$ (d. $J=7.0 \mathrm{~Hz}, \mathrm{lH}) .3 .87$ (d. $J=9.4$ Hz. $1 \mathrm{H}), 3.78$ (d. $J=9.4 \mathrm{~Hz}, 1 \mathrm{H}) .2 .95(\mathrm{dd}, J=13.4 .8 .0 \mathrm{~Hz}$, IH). $2.49(\mathrm{dd}, J=13.4,6.6 \mathrm{~Hz}, 1 \mathrm{H}):{ }^{13} \mathrm{C}$ NMR (DMSO- $\left.d_{6}\right) \delta$ 165.81, 156.51. 145.68. 144.87, 142.60, 140.82. 124.42, 93.57. 69.51. 60.67. 48.90, 42.77. Anal. Calcd. for $\mathrm{C}_{12} \mathrm{H}_{15} \mathrm{~N}_{3} \mathrm{O}_{2} \cdot 0.5 \mathrm{MeOH}: \mathrm{C}, 60.22 ; \mathrm{H} .6 .86 ;$ N. 16.82. Found: C. $60.33: \mathrm{H}, 6.98 ; \mathrm{N}, 16.90$.

(rel)-(1'R,t'R)-9-[t-(Hydroxymethy])-t-viny]-cyclopent2-en-1-yl] 2-amino-6-chloropurine (19): Purine nucleoside analogue 19 was prepared from compound 16 using a similar procedure to that described for compound 17: yield 69\%: mp 178-180 ${ }^{\circ} \mathrm{C}$; ${ }^{1} \mathrm{H}$ NMR (DMSO- $d_{6} .300 \mathrm{MHz}$ ) $\delta$ 10.71 (br s, lH), 7.87 (s. lH), 6.51 (br s, $2 \mathrm{H}$ ). 6.41 (dd. $J=$ $5.4 .1 .2 \mathrm{~Hz}, 1 \mathrm{H}) .6 .22-6.10(\mathrm{~m} .4 \mathrm{H}) .5 .78(\mathrm{~d} . J=6.4 \mathrm{~Hz}, 1 \mathrm{H})$. 5.01 (t. $J=5.2 \mathrm{~Hz}, 1 \mathrm{H}) .3 .89(\mathrm{~d}, J=9.6 \mathrm{~Hz}, 1 \mathrm{H}), 3.78(\mathrm{~d} . J=$ $9.4 \mathrm{~Hz}, 1 \mathrm{H}), 2.88$ (dd. $J=13.4 .8 .2 \mathrm{~Hz}, 1 \mathrm{H}) .2 .45(\mathrm{dd} . J=$ 13.4. $6.8 \mathrm{~Hz}, 1 \mathrm{H}):{ }^{13} \mathrm{C}$ NMR (DMSO- $\left.d_{2}\right) \delta 160.02,155.21$, 152.98, 146.21. 143.43. 141.98, 140.72, 133.21. 124.59, 70.55. 61.32, 47.65. 43.65: Anal Calcd for $\mathrm{C}_{12} \mathrm{H}_{1+} \mathrm{ClN}_{5} \mathrm{O}$ 1.0 $\mathrm{H}_{2} \mathrm{O}:$ C. $50.40 ;$ H. 5.20; N. 22.61. Found: C, $50.50 ; \mathrm{H}$, $5.18 ; \mathrm{N}, 22.53$.

(rel)-(1'R,4'R)-9-[4-(Hydroxymethyl)-4-viny]-cyclopent2-en-1-yl] guanine (20). Mercaptoethanol $(0.18 \mathrm{~mL}, 2.58$ mimol) and NaOMe (2.0 mL, 2.0 mmol. $1.0 \mathrm{M}$ solution in $\mathrm{MeOH})$ was added to a solution of compound 19 (128 mg. $0.44 \mathrm{mmol})$ in $\mathrm{MeOH}(10 \mathrm{~mL})$. and refluxed overnight After cooling, the reaction mixture was neutralized with a few drops of glacial $\mathrm{AcOH}$ and concentrated under reduced pressure. The residue was purified by silica gel column 
chromatography (MeOH/C $\left.\mathrm{CH}_{2} \mathrm{Cl}_{2} .1: 5\right)$ to give the carbovir analogue $20(72 \mathrm{mg} .60 \%)$ as a solid: $\mathrm{mp} 186-188^{\circ} \mathrm{C}$. UV $\left(\mathrm{H}_{2} \mathrm{O}\right) \hat{\lambda}_{\max } 253.0 \mathrm{~cm} ;{ }^{1} \mathrm{H}$ NMR (DMSO- $\left.d_{6}, 300 \mathrm{MHz}\right) \delta$ 10.75 (br s, lH), $7.98(\mathrm{~s}, \mathrm{lH}), 6.50$ (br s. $\mathrm{lH}) .6 .40(\mathrm{~d} . J=5.2$ $\mathrm{Hz}, 1 \mathrm{H}), 6.13-6.01(\mathrm{~m}, 4 \mathrm{H}), 5.72(\mathrm{dd}, J=6.6 .1 .4 \mathrm{~Hz}, \mathrm{lH})$. $4.98(\mathrm{t} . J=5.2 \mathrm{~Hz}, \mathrm{IH}), 3.88(\mathrm{~d} . J=9.2 \mathrm{~Hz}, \mathrm{lH}) .3 .74(\mathrm{~d}, J=$ $9.4 \mathrm{~Hz} .1 \mathrm{H}), 2.89$ (dd. $J=13.2 .8 .4 \mathrm{~Hz}, 1 \mathrm{H}) .2 .47$ (dd, $J=$ $13.4,6.6 \mathrm{~Hz} . \mathrm{lH}$ ) $;{ }^{13} \mathrm{C}$ NMR (DMSO- $d_{3}$ ) $\delta 157.84,154.70$. 152.29. 145.67, 142.51. 140.98, 136.40. 132.28, 117.39 . 70.67. 61.39, 48.11, 42.97; Anal Calcd. for $\mathrm{C}_{13} \mathrm{H}_{15} \mathrm{~N}_{5} \mathrm{O}=1.0$ $\mathrm{H}_{2} \mathrm{O}$ : C. $53.60:$ H. $5.88 ;$ N. 24.04. Found: C. $53.52 ; \mathrm{H}, 5.79$ : N. 24.48 .

\section{References}

1. (a) O-Yang. C.: Wu. H. Y.: Fraser-Smith. E. B.: Walker. K. A. M. Tetrahedron Lett. 1992. 33. 37. (b) Maag. H.: Nelsonl. J. T.: RiosSteiner. J. L.: Prisle. E. J. J. Hed Chent 1994. 37. 431. (c) Haraguchi. K: Takeda, S.: Tanaka. H: Nitanda, T: Baba, M.: Dutschman, G. E.: Cheng, Y.-C. Bioorg. Med. Chem. Lett. 2003. 13,3775. (d) Kunnamoto. H.: Nakai, T.: Haraguehi. K.: Nakamura. K. T.: Tanaka. H.: Baba. M.: Cheng. Y.-C. J. Med Chem, 2006. 49.7861 .

2. Sugimoto. I.: Shuto. S.: Mori. S.: Shigeta. S.: Matuda. A. Bioorg. Hed. Chem. Lett. $1999,9,385$.

3. Nomura, M.: Shuto, S.: Tanaka. M.: Sasaki. T.: Mori. S :- Shigeta. S.: Matuda. A. J. Ifed Chem. 1999. 42. 2901.
4. (a) Huyn. D. M.: Okabe. M. Chent. Rev. 1992. 92. 1745. (b) Crimmins. M. T. Tetrahedron 1998. 5t. 9229. (c) Ariona. O.: Gómez. A. M.: López. J. C.: Plumet. I. Chem. Rev 2007. 107. 1919.

5. Ueland. P. M. Phamacol Rev 1982. 34. 223.

6. (a) Vince, R.: Hua, M: Brownell. J; Daluge. S.; Lee. F. C: Shan110n. W. M.: Lavelle. G. C.: Qualls. J.: Weisolw. O. S.: Kiser. R Biochem. Biophns. Res Commm. 1988. 156. 1046. (b) Vince. R.: Hua. M. J. Med Chen. 1990. 33. 17. (c) Vince. R. Nicleic Acids Symp. Ser. 1991, 25. 193

7. Symonds, W. Cutrell. A.; Edwards. M.: Steel. H: Spreen. B: Powell. G.: MoGuirk, S.: Hetherington. S. Clin. Ther 2002. 24. 565 .

8. (a) Nugiel. D. A.: Takobs. K.: Kaltenbach. R. F.: Worley. T.: Patel. M.: Mever. D. T.: Jadhay. P. K.: De Lucca. G. V: Smyser. T. E.: Klabe. R. M: Bacheler. L. T.: Rayner. M. M.: Seitz, S. P. J. Med. Chem 1996. 39.2156. (b) Nahm. S.: Weinreb, S. M. Temahedron Lett. $1981,22.3815$.

9. Honn. T. H.: Ko. O. H. Bull. Korem Chem. Soc. 2003. 24. 1289.

10. (a) Ziegler. F. E. Chem. Rev 1988. 88. 1423. (b) Hong. J. H.: Gao. M. Y.: Chu. C. K. Tetrahedron Lett. 1999. 40.231. (c) Hong. J. H.: Lee. K.; Choi, Y.: Chu. C. K. Tetrahedron Lett. 1988, 39.3443 . (d) Oh. C. H.: Hong. J. H. Bull. Koram Chem. Soc. 2005 26. 1520.

11. (a) Grubbs. R. H.; Miller. S. J. Acc. Chem. Res. 1995. 28. 446. (b) Grubbs. R. H.: Chang. S. B. Tetrohedron 1998. 5t. 4413.

12. (a) Trost. B. M.: Kallander. L. S. J. Org. Chem. 1999. 64. 5427 (b) Trost. B. M.: Shi. Z. J. An Chem. Soc. 1996. 118. 3037 .

13. Jeong, L. S.; Kim, H. O.: Moon, H. R.: Hong. J. H.; Yoo. S. J.; Choi. W. J.; Chun. M. W. Lee C. K. J. Hed. Chem. 2001. Ht. 806. 